\title{
Comparison of rhizosphere microbial communities under Masson pine families with different carbon sequestration abilities
}

\author{
Fan $\mathrm{Wu}^{1}$, Xiaobo $\mathrm{Sun}^{1}$, Xingfeng $\mathrm{Hu}^{1}$, Peihuang Zhu ${ }^{1}$, Bingzhang Zou ${ }^{2}$, Nengqing Lin ${ }^{2}$, \\ Jingquan $\operatorname{Lin}^{2}$, and Kongshu $\mathrm{Ji}^{1}$ \\ ${ }^{1}$ Nanjing Forestry University \\ ${ }^{2}$ Baisha State-Owned Forest Farm
}

September 16, 2020

\begin{abstract}
To compare the rhizosphere communities under Masson pine trees with different carbon sequestration abilities, we sampled three families of Masson pine that showed significant differences in their carbon sequestration ability and conducted high-throughput sequencing of the 16S rRNA and ITS in the corresponding soil samples. The diversity of rhizosphere soil microorganisms, the patterns of differences in relative abundance among the different samples and the responses of microorganisms to environmental factors were analyzed. There was no significant difference in the diversity of soil bacteria or fungi among the different samples from Masson pine families with different carbon sequestration abilities, but with the increase in carbon sequestration, the dominant phyla of bacteria and fungi changed. The dominant phylum of soil bacteria changed from Proteobacteria to Acidobacteria, and that of fungi changed from Ascomycota to Basidiomycota. In addition, we investigated the core microbial communities at the genus level among the different samples, and a significant portion of the core genera were involved in carbon metabolism. The microbial communities were greatly influenced by environmental factors such as soil carbon content, soil moisture and altitude. Soil fungi were more sensitive than soil fungi to the rhizosphere activity of Masson pine.
\end{abstract}

\section{KEYWORDS}

Rhizospheric microorganisms, Masson pine, Bacteria, Fungus, Environmental factors

\section{1 | INTRODUCTION}

According to data from the Global Forest Resources Assessment (IPCC, 2013), the total amount of carbon sequestered by global forests is as high as 1950-3150 Pg C each year; the carbon sequestration by vegetation reaches 450-650 Gt, accounting for approximately $43.5 \%$ of the total. On the other hand, Schlesinger (1990) noted that soil is the largest reservoir of carbon in terrestrial ecosystems, storing two-thirds of their organic carbon. The rhizosphere microecosystem is the link between plants, soil and microorganisms, the most active part of the global carbon biochemical cycle, and the focus of research on the systematic mechanisms of the global carbon cycle (Schweinsberg-Mickan, Jorgensen, \& Muller, 2012; Carrillo, Dijkstra, Pendall, LeCain, \& Tucker, 2014). Photosynthetic products are transported from leaves to various tissues, such as root tissues, for storage, and this transport occurs through material flow caused by pressure differences. The remaining photosynthetic products are released into the surrounding soil by the plant root system as various organic and inorganic compounds that form rhizodeposits. In addition, carbon that enters the soil through a series of biochemical processes is circulated and redistributed among roots, the soil and microorganisms to maintain the balance of rhizosphere carbon absorption and release (Jones, Nguyen, \& Finlay, 2009).

Studies have shown that carbon sequestration by plants is particularly important for rhizosphere microorganisms. Over $40 \%$ of the complex carbon produced by photosynthesis enters rhizosphere soil through 
plant roots to nourish microorganisms and maintain their normal metabolic functions (Rodriguez, et al., 2019). Cheng et al. (1996) noted that root exudates, as carbon sources for microbial utilization, increase respiration by rhizosphere microorganisms. The differences in carbon utilization efficiency among microbial communities mainly depend on the differences in their microbial functional families related to carbon source utilization $(\mathrm{Xu}, 2012)$. Yin et al. (2018) found that increasing the atmospheric $\mathrm{CO}_{2}$ concentration to promote carbon metabolism in Kandelia candel did not significantly increase the abundance of the rhizosphere bacterial community. Moreover, Xiao et al. (2017) noted that carbon sequestration by Bothriochloa ischaemumsignificantly increased the contents of total PLFAs (phospholipid-derived fatty acids) and fungal PLFAs in rhizosphere soil. In addition, Feng et al. (2011) indicated that under increased carbon metabolism conditions in rice, the proportions of aerobic, anaerobic and phototrophic bacteria in the bulk soil increased (from $0.5 \%$ to $1.5 \%$ ), while no significant effects were observed in rhizosphere soil. It has been noted that among rhizosphere microorganisms, the response of fungi to photosynthetic carbon sequestration by plants is clearer than that of bacteria $(\mathrm{Xu}, 2012)$ because fungal mycelia can accelerate the turnover cycle of the fungal carbon metabolism (which takes approximately one week), while bacteria generally need more than two weeks to turn over carbon (Ostle, et al., 2003; Staddon,Ramsey, Ostle, Ineson, \& Fitter, 2003).

Masson pine (Pinus massoniana) is a large perennial tree that is widely distributed in 17 provinces and autonomous regions in the southern Qinling Mountains in China (Wu, et al., 2020). Masson pine thrives in light, is shade intolerant and prefers a warm and humid climate. It can grow in red soil, gravel soil and sandy soil and is used as a pioneer tree species for forest restoration in barren mountainous areas (Wang, et al., 2019). Previous studies have shown that Masson pine has a high carbon sequestration ability. Elisa et al. (2003) showed that the carbon sequestration in Masson pine organs ranged from 533.93 to $568.08 \mathrm{~g} \cdot \mathrm{kg}^{-1}$, which is higher than the carbon contents of 32 common tropical tree species (444.0-494.5 $\mathrm{g} \cdot \mathrm{kg}^{-1}$ ). The carbon sequestration ability of plants directly affects the quantity of root exudates (Ainsworth, 2008). However, the response of soil microbial communities, particularly rhizosphere microorganisms, to plant carbon sequestration has rarely been studied, especially under Masson pine. In this study, based on Masson pine from different families, samples with significant differences in carbon sequestration ability were selected as experimental materials. The corresponding rhizosphere soil was obtained for 16S rRNA and ITS sequencing to analyze the differences in in the number and taxonomic diversity of bacteria and fungi and their patterns in response environmental factors. This research provides guidance toward further understanding the response of microorganisms to plant carbon sequestration, which will be helpful in predicting the effects of climate change on rhizosphere microbial communities.

\section{$2 \mid$ MATERIAL AND METHODS}

\subsection{Study site}

This study was conducted in the progeny test plantation of the Masson pine seed orchard at the Baisha StateOwned Forest Farm $\left(25^{\circ} 15^{\prime} \mathrm{N}, 116^{\circ} 62^{\prime} \mathrm{E}\right)$, Shanghang County, Fujian Province. The samples from the forests were collected in 2001, and the experimental trees were planted in 2003. There were 68 families (Kang, 2012). Before the experiment was carried out, it was found that due to human activities, the number of samples in some families did not meet the requirements for statistical analysis. Therefore, given the situation, 24 families were selected as the experimental families (Table S2).

\subsection{Estimation of carbon storage in different Masson pine families}

To avoid destroying trees, regression equations were used to estimate the average carbon sequestration by each family. Approximately 30 individuals in each family were randomly selected as experimental samples, and the height and DBH (diameter at breast height) of each sample were measured. The regression equations in professional standards released by China's Forestry Administration (Cai, et al., 2014) were used to estimate the biomass of each organ (including the trunk, branches, leaves, bark, and roots) based on the tree height and DBH. Then, the biomass of each organ was multiplied by the corresponding carbon coefficient (trunk: 0.5186 , branches: 0.5174 , leaves: 0.5785 , bark: 0.4994 , and roots: 0.5082 ) to obtain the total carbon sequestration. 
The total carbon sequestered by a single tree was obtained by adding the carbon sequestered in each organ. The mean value of all samples from the same family was used as an index to evaluate the carbon sequestration level of the family. The relevant regression equations are provided in Table S1.

\section{3 | Soil sampling}

The families with high, low and intermediate carbon sequestration were selected for follow-up experiments. Three individuals with carbon sequestration values that were close to the mean value for each experimental family were selected as the samples. Five sampling points near each sample were chosen, and $5^{\sim} 10 \mathrm{~cm}$ bulk soil was dug up. The roots were carefully pulled out of the soil with a shovel, and the loosely attached soil was gently shaken off. The rhizosphere soil was closely attached to the roots. Litter and humus were removed from the soil surface before soil sampling, and the rhizosphere soil from the five sites near each sample tree was mixed together to form a composite soil sample. The soil samples were divided into two parts; one part was placed into a $5 \mathrm{ml}$ freezing tube and immediately frozen in dry ice for sequencing, and the other part was loaded into a $50 \mathrm{ml}$ centrifuge tube for analyses of soil physical and chemical properties.

\subsection{Detection of the physical and chemical properties of rhizosphere soil}

The rhizosphere soil total organic carbon was determined by the combustion oxidation nondispersive infrared absorption method according to the Chinese Environmental Protection Standard (Jian, Zhai, Wang, \& Cai, 2020). The experimental temperature was set at $900^{\circ} \mathrm{C}$, and the oxygen pressure was $900 \mathrm{Mbar}$. The gas flow rate in the analysis module was $150-165 \mathrm{~mL} \cdot \mathrm{min}^{-1}$. The soil total nitrogen was determined based on the Kjeldahl method as provided in the Chinese Environmental Protection Standard (Zhang, et al., 2015). To determine the rhizosphere soil $\mathrm{pH}, 5 \mathrm{~g}$ soil samples were ground and passed through a 100 mesh sieve, and

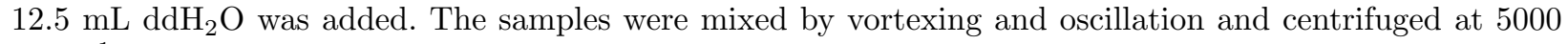
$\mathrm{r} \cdot \mathrm{min}^{-1}$ for $5 \mathrm{~min}$, and the supernatant was separated and directly tested with a $\mathrm{pH}$ meter. To determine the rhizosphere soil moisture, soil samples $(20 \mathrm{~g})$ were weighed and dried at $105^{\circ} \mathrm{C}$ for $6 \mathrm{~h}$. After cooling to room temperature and weighing again, the difference between the two weights was divided by the fresh soil weight $(20 \mathrm{~g})$ to obtain the moisture content.

\subsection{DNA extraction, high-throughput sequencing and analysis}

The FastDNA ${ }^{\mathrm{TM}}$ Spin Kit for Soil (MP Biomedicals, California, USA) was used to extract DNA from 0.5 $\mathrm{g}$ soil samples. The operation process was performed in strict accordance with the instruction manual. The barcode primers 515F (5'-GTGCCAGCMGCCGCGG-3') and 907R (5'-CCGTCAATTCMTTTRAGTTT3') were used to amplify the bacterial $16 \mathrm{~S}$ rDNA. ITS1F (5'-CTTGGTCATTTAGAGGAAGTAA-3') and ITS2R (5'-GCTGCGTTCTTCATCGATGC-3') were used to amplify the fungal ITS sequence. The PCR amplification procedure was carried out according to the instructions for TransStart ${ }^{(\mathrm{r})}$ FastPfu DNA Polymerase (TransGen Biotech, Beijing). Each sample was subjected to PCR three times. The PCR products from the same sample were mixed and detected with $2 \%$ agar-gel electrophoresis, recovered using the AxyPrepDNA gel recovery kit (Axygen Biosciences, CA, USA), and purified using the agar-gel DNA purification kit (TransGen Biotech, Beijing). The purified PCR products were sequenced according to the default parameters on the MiSeq PE300 platform. The original data were stored in the NCBI Sequence Read Archive database (accession number PRJNA662187 for bacteria and PRJNA662212 for fungi).

The raw sequence data were analyzed and quality-controlled using fastp (version 0.19.6, https://github.com/OpenGene/fastp). Bioinformatics statistical analysis was performed using Usearch (version 7.0, http://drive5.com/uparse/) for OTUs (operational taxonomic units) at 97\% similarity. The OTUs were subsampled according to the minimum sample sequence number $(35,000)$. The taxonomic analysis of the OTU representative sequences was carried out by the RDP classifier Bayesian algorithm (version 2.2, http://sourceforge.net/projects/rdp-classifier/, the default confidence threshold value was 0.7 ), and the community composition of each sample was counted at different taxonomic levels. The Silva bacterial 16S comparison database and the Unite fungal ITS comparison database were used. 


\section{6 | Statistical analyses}

A Venn diagram of the microbial community diversity was constructed with the $\mathrm{R}$ Venn diagram package (Chen, \& Boutros, 2011) based on the common and unique OTUs in the different samples. The Chao and Shannon index of $\alpha$-diversity were calculated according to the corresponding formulas shown in Table S1. The relative abundance histogram at the phylum level was plotted using the $\mathrm{R}$ ggplot2 package (Kahle, \& Wickham, 2013) based on the data sheet in the tax_summary_a folder. Welch T-tests were conducted to determine the significance of differences among families (Garcia-Lledo, Vilar-Sanz, Trias, Hallin, \& Baneras, 2011). The Bonferroni method was used to conduct multiple test corrections to evaluate the significance level of taxonomic abundance differences and to identify the significantly different phyla among samples. For the core microbiome analyses at the genus level, common OTUs with relative richness values higher than $1 \%$ were extracted from different samples for graphical purposes. Pie and box charts were generated with the R ggplot2 package and SPSS 16.0, respectively (Perez-Jaramillo, et al., 2019). RDA (redundancy analysis) was used to clarify the relationships between soil physicochemical properties and the rhizosphere microbial community using the $\mathrm{R}$ vegan package ( $\mathrm{Ng}$, et al., 2014). Correlation heatmap analysis was performed by calculating the Pearson correlation coefficients between the environmental factors and the selected taxa and drawing the heatmap diagram using the R Pheatmap package. One-way ANOVA and Duncan multiple comparison tests were conducted using SPSS 16.0.

3

\section{RESULTS}

\subsection{Comparison of carbon sequestration among families}

The height, DBH and carbon sequestration data for individuals are shown in Table 1 and Table S2. Family No. 38 had the greatest mean tree height at $14.15 \mathrm{~m}$; this was 1.22 times the lowest mean height $(11.54 \mathrm{~m})$, which was observed in family No. 21. family No. 66 had the largest mean DBH $(12.20 \mathrm{~cm})$; this was 1.16 times the lowest mean DBH, which was observed in family No. $4(10.49 \mathrm{~cm})$. family No. 66 had the highest mean carbon sequestration value at $28.245 \mathrm{~kg}$; this was 1.45 times the lowest mean carbon sequestration, which was observed in family No. $4(19.501 \mathrm{~kg})$. Duncan's multiple comparison results showed that there were significant differences in tree height, DBH and carbon sequestration among the different families. By considering the data from all the three indicators, families No. 4, No. 64 and No. 66 were selected as the representative families showing low, medium and high carbon sequestration for the subsequent experiments.

TABLE 1 Carbon sequestration statistics for the samples from different families

\begin{tabular}{lllllll}
\hline $\begin{array}{l}\text { Family } \\
\text { Number }\end{array}$ & Sample Size & Min & Max & Mean & SD & Duncan \\
1 & & & & & \\
4 & 29 & 9.674 & 49.532 & 20.360 & 8.053 & efg \\
5 & 27 & 7.960 & 40.340 & 19.501 & 8.460 & g \\
9 & 31 & 11.221 & 53.197 & 23.740 & 10.031 & abcdefg \\
12 & 31 & 10.838 & 36.815 & 19.727 & 7.026 & fg \\
13 & 30 & 10.527 & 46.036 & 22.728 & 8.023 & bcdefg \\
15 & 32 & 8.097 & 55.123 & 20.991 & 9.001 & defg \\
21 & 29 & 12.886 & 43.415 & 27.451 & 7.976 & ab \\
25 & 32 & 14.657 & 42.851 & 25.984 & 8.176 & abcd \\
27 & 31 & 12.387 & 52.871 & 22.724 & 8.463 & bcdefg \\
29 & 30 & 17.823 & 49.459 & 27.733 & 7.894 & ab \\
33 & 31 & 11.313 & 43.143 & 25.527 & 7.639 & abcde \\
34 & 28 & 3.897 & 42.279 & 25.158 & 9.845 & abcde \\
38 & 32 & 11.174 & 46.091 & 27.157 & 7.761 & abc \\
& 32 & 14.665 & 45.641 & 24.676 & 6.951 & abcdefg
\end{tabular}




\begin{tabular}{lllllll}
39 & 31 & 2.533 & 37.873 & 20.966 & 6.426 & defg \\
40 & 31 & 11.861 & 50.361 & 24.974 & 8.259 & abcdef \\
44 & 32 & 4.397 & 54.755 & 26.665 & 12.228 & abc \\
49 & 29 & 10.645 & 35.901 & 23.869 & 7.097 & abcdefg \\
51 & 28 & 7.925 & 35.535 & 19.776 & 6.960 & fg \\
54 & 33 & 7.264 & 41.909 & 22.186 & 8.279 & cdefg \\
64 & 30 & 13.026 & 42.476 & 24.475 & 7.614 & abcdefg \\
66 & 26 & 13.026 & 56.274 & 28.245 & 9.575 & a \\
67 & 27 & 10.096 & 41.002 & 21.291 & 9.190 & defg \\
68 & 29 & 10.742 & 47.786 & 24.848 & 9.824 & abcdef \\
\hline
\end{tabular}

Note : The corresponding unit of data in the table is $\mathrm{kg}$

Abbreviations: SD, standard deviation; Duncan, Duncan multiple comparisons.

\section{$3.2 \mid$ Comparison ofrhizosphere soil properties}

Table 2 shows that there were no significant differences in $\mathrm{pH}$, total nitrogen content or $\mathrm{C} / \mathrm{N}$ among the three families. The total organic carbon content and soil moisture in the samples increased with increasing carbon sequestration, and the differences among the three families were significant $(\mathrm{P}<0.05)$. In terms of altitude, the mean values of families No. 4 and No. 64 were significantly higher than that of No. 66. Combining these data with those in Table 1, we speculated that the growth and carbon sequestration capacity of samples were directly proportional to their soil moisture and total organic carbon but inversely proportional to their altitude.

TABLE 2 Properties of the rhizosphere soils of the different families

\begin{tabular}{llll}
\hline & Family No.4 (Low) & Family No.64 (Middle) & Family No.66 (High) \\
$\mathrm{pH}$ & $4.55 \pm 0.42 \mathrm{a}$ & $4.97 \pm 0.35 \mathrm{a}$ & $4.95 \pm 0.12 \mathrm{a}$ \\
Total organic Carbon $\left(\mathrm{C}, \mathrm{g} \cdot \mathrm{Kg}^{-1}\right)$ & $14.17 \pm 1.69 \mathrm{c}$ & $16.55 \pm 0.9 \mathrm{~b}$ & $21.78 \pm 1.78 \mathrm{a}$ \\
Tatal Nitrogen (N, g. Kg & $0.77 \pm 0.13 \mathrm{a}$ & $0.86 \pm 0.07 \mathrm{a}$ & $1.2 \pm 0.36 \mathrm{a}$ \\
$\mathrm{C} / \mathrm{N}$ & $18.40 \pm 1.98 \mathrm{a}$ & $19.24 \pm 0.64 \mathrm{a}$ & $18.15 \pm 1.32 \mathrm{a}$ \\
Soil Moisture (SM, \%) & $5.67 \pm 0.25 \mathrm{~b}$ & $8.53 \pm 0.67 \mathrm{a}$ & $7.7 \pm 0.62 \mathrm{a}$ \\
Altitude (AL, m) & $467.67 \pm 5.86 \mathrm{a}$ & $476.67 \pm 5.77 \mathrm{a}$ & $453 \pm 3.61 \mathrm{~b}$ \\
\hline
\end{tabular}

\subsection{Diversity analysis of the rhizosphere microbial community}

A total of 1,079,408 and 1,675,540 high-quality sequences were analyzed for bacteria and fungi, respectively. In bacteria, the high, medium and low carbon sequestration rhizosphere soils contained 1704, 1641, and 1672 OTUs, respectively. A total of 1221 OTU sequences were common among the three kinds of samples (Figure $1 \mathrm{~A})$. In contrast, 808, 654 and 838 fungal OTUs were identified from the high, medium and low carbon sequestration rhizosphere soil samples, respectively. Only 254 soil fungal OTU sequences were common among the three sample types (Figure $1 \mathrm{~B}$ ). B). The OTUs of fungi in each sample were far fewer than those of bacteria.

The Chao and Shannon index of bacteria and fungi are shown in Figure 1. C,D. For bacteria, the Chao index of the different samples fluctuated between 1476 and 1521, and samples with high carbon sequestration had slightly higher values that were not significantly different from those of the other samples. The Chao index for fungi was approximately 400. The difference in the Chao index values between fungi and bacteria was significant (Figure 1C). On the other hand, the Shannon index for bacteria and fungi from the different families showed only slight differences, and the differences among samples were not significant (Figure 1D). These results indicate that overall, combined with the other parameters used to evaluate the microbial community $\alpha$-diversity shown in Table S3, there was little difference in $\alpha$-diversity among the rhizosphere 
microorganisms in the various samples.
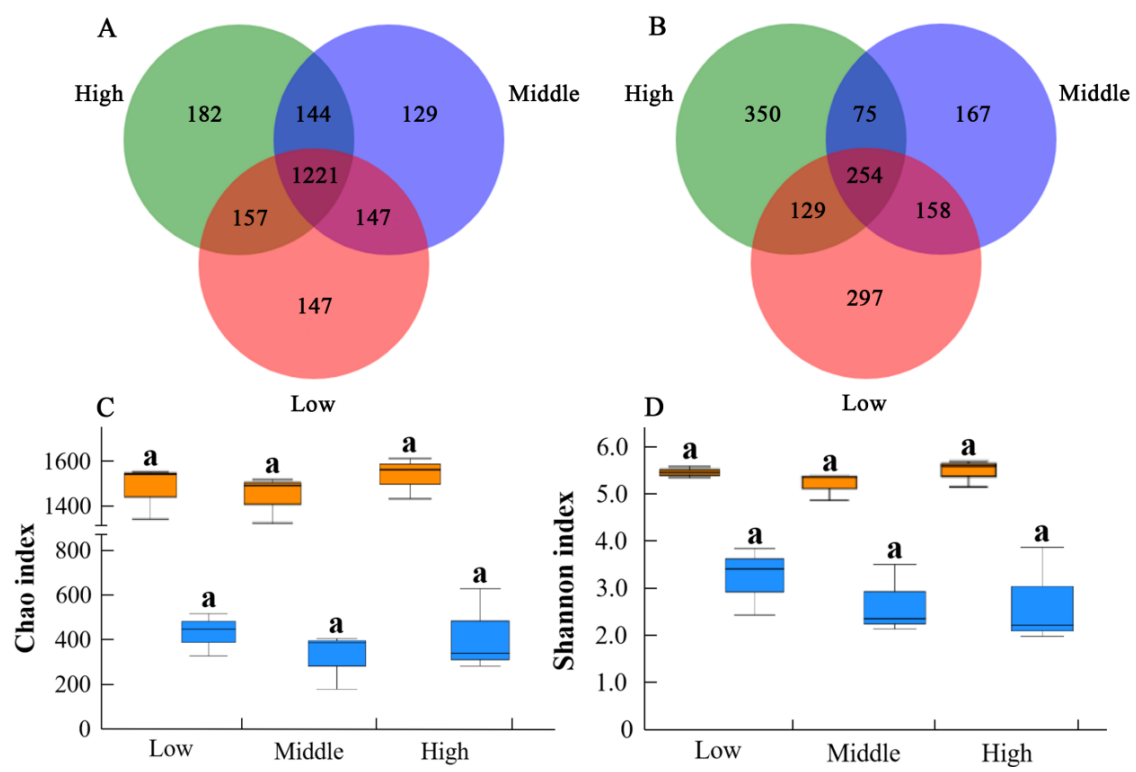

FIGURE 1 Rhizosphere microbial $\alpha$-diversity of the different samples. A-B, Bacterial and fungal OTU Venn diagrams, respectively; C, Chao index; D, Shannon index. Orange and blue represent bacteria and fungi, respectively.

\section{4}

\section{Composition of rhizosphere soil bacterial and fungal communities}

The composition of bacterial and fungal communities at the phylum level is shown in Figure S1. Six bacterial phyla with relative abundances higher than $1 \%$ were detected, namely, Acidobacteria, Proteobacteria, Chloroflexi, Actinobacteria, Planctomycetes and Bacteroidetes. Four fungal phyla with relative abundances higher than 1\% were detected, namely, Basidiomycota, Ascomycota, Mucoromycota, and one unidentified phylum (Unclassified_k_Fungi). Based on the above bacterial and fungal data, taxonomic differences in the rhizosphere microbial communities of the different samples were analyzed (Figure 2, Table S3-S8). In terms of the bacteria, the relative abundance of Acidobacteria increased with increasing carbon sequestration ability. The difference in its abundance between the medium and high families was not significant, while the differences between the medium and low samples and the high and low samples were significant. The relative abundance of Proteobacteria declined with increasing carbon sequestration ability. There was no significant difference in its abundance between the middle and high samples, while the differences between the medium and low samples and the high and low samples were significant. The other phyla showed no significant differences among the three samples. This indicated that the carbon sequestration process in Masson pine might have a positive effect on Acidobacteria and a negative effect on Proteobacteria. In terms of the fungi, the relative abundances of Basidiomycota and Unclassified_k_Fungi increased with increasing carbon sequestration ability. The relative abundances of Ascomycota and Mucoromycota decreased with increasing carbon sequestration, but in general, the relative abundance of the four main fungi did not show significant differences among the different samples. 


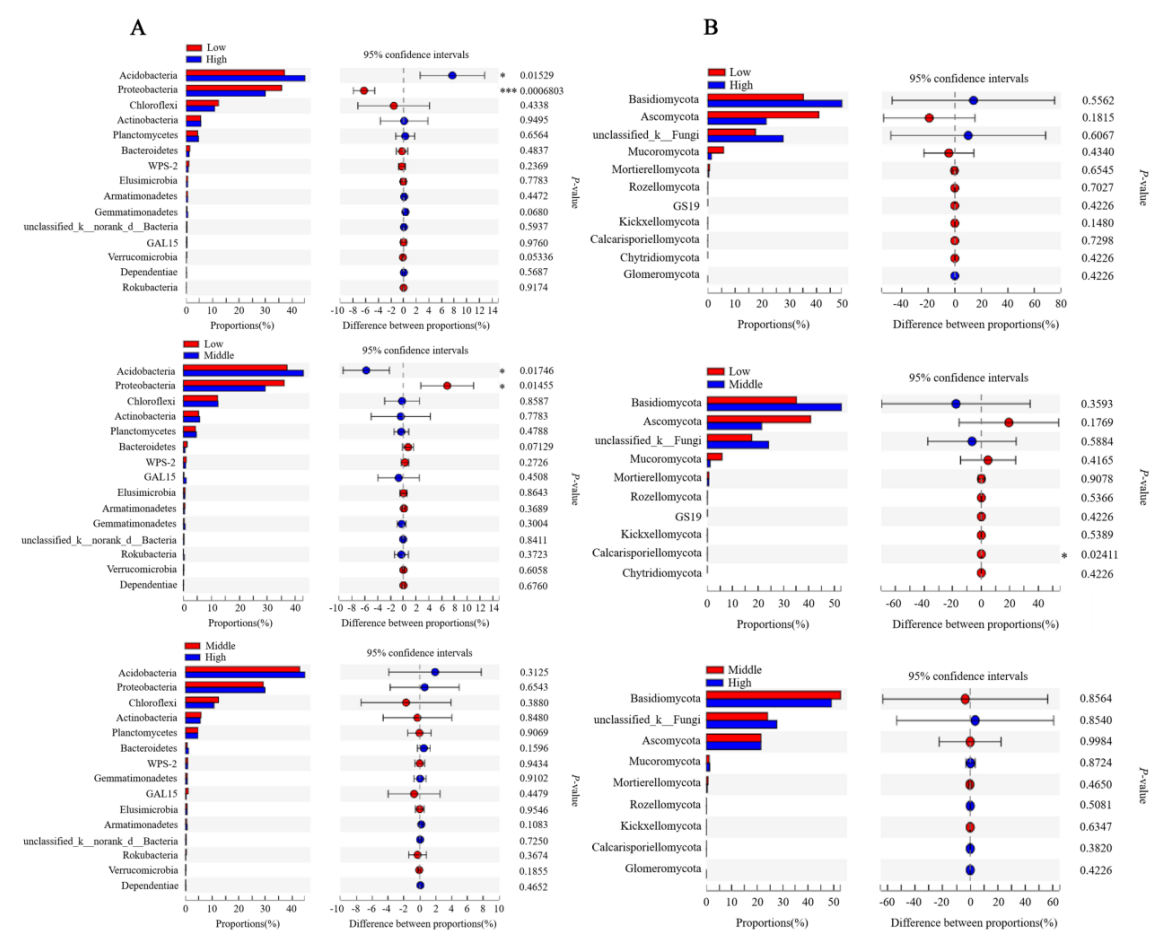

FIGURE 2 Differential abundance of bacterial (A) and fungal (B) OTUs in the rhizosphere soil of different samples (at the phylum level). The Welch T-test was used for statistical analysis. The Bonferroni method was used for multiple hypothesis test correction. '*': $\mathrm{P}<0.05$, '***': $\mathrm{P}<0.01$.

\subsection{Core microbial community}

As shown in Figure 3, 5,017 bacterial OTUs were detected in the three families. Among them, 1,221 OTUs were found in all samples, accounting for $24.34 \%$ of all OTUs. OTUs with an abundance of more than $1 \%$ were selected as representative of the core bacterial family. These OTUs were assigned to various genera. Within Acidobacteria, norank_Subgroup2 accounted for the largest proportion of the genera (containing 12 OTUs, with a relative abundance of $75.32 \%$ ), followed by norank_Acidobacteriales (3 OTUs, relative abundance 14.00\%). Acidibacter (4 OTUs, relative abundance 34.19\%), norank_Xanthobacteraceae (2 OTUs, relative abundance 19.85\%), Burkholderia-Caballeronia-Paraburkholderia (2 OTUs, relative abundance 19.14\%), and Bradyrhizobium (1 OTU, relative abundance 8.91\%) were the most abundant in Proteobacteria, and HSB_OF53-F07 was the most abundant in Chloroflexi (1 OTU, relative abundance 2.90\%). In the box plots corresponding to each bacterial genus, the abundances of Acidothermus and Bryobacter in Acidobacteria showed a trend of leveling off or decreasing first and then increasing, respectively, with increasing carbon sequestration. However, the relative abundance of each genus in Proteobacteria and Chloroflexi decreased to different degrees with the increase in carbon sequestration in the samples (Figure 3).

A total of 2,300 fungal OTUs were detected in the three families. Among them, 254 OTUs were found in all samples, accounting for $11.04 \%$ of all fungal OTUs. According to the bacterial classification method, these OTUs belonged to unclassified_Basidiomycota (1 OTU, relative abundance 33.74\%), Russula (1 OTU, relative abundance 26.36\%), Saitozyma (1 OTU, relative abundance 19.30\%) and Geminibasidium (1 OTU, relative abundance 19.16\%) in Basidiomycota. Unclassified_Ascomycota (4 OTUs, relative abundance 37.91\%), Penicillium (1 OTU, relative abundance $32.25 \%$ ), Archaeorhizomyces (2 OTUs, relative abundance 14.32\%), Oidiodendron (1 OTU, relative abundance 6.70\%), and unclassified_Helotiales (1 OTU, relative abundance 4.89\%) were the most abundant genera in Ascomycota, and Umbelopsis (1 out, relative abundance 4.38\%) was the most abundant in Mucoromycota. In the box plots, in Basidiomycota, the relative abundance of all genera 
exceptSaitozyma showed an increasing trend with the increase in carbon sequestration. However, the relative abundances of fungi in Ascomycota and Mucoromycota showed a continuously decreasing trend except that the relative content of Penicillium decreased first and then increased (Figure S2).

FIGURE 3 Bacterial communities in the core rhizosphere of different samples. The inner (various colors) and outer (yellow) pie charts represent the core bacterial phyla and genera, respectively. The size of each phylum and genus in the pie chart represents their contribution to the overall abundance. The box plots show the relative abundance of the corresponding genus in the different samples. Some genera appeared in the taxonomy database with no corresponding scientific name and are labeled 'norank'.

\section{6 $\mid$ Relationships between the rhizosphere microbial community and soil environmental fac- tors}

The RDA results show that for bacteria, the first axis explained $53.97 \%$ of the variation in the data, and the second axis explained $14.06 \%$, representing a cumulative explanatory power of $68.03 \%$ (Figure $4 \mathrm{~A}$ ). The first two axes reflected the relationships between the genera and their influencing factors well. As shown in Figure 4A, norank_Subgroup 2 had the strongest correlation with environmental factors, whilenorank_Gemmataceae had the weakest correlation. On the other hand, Acidothermus was greatly affected by the total organic carbon, total nitrogen content and $\mathrm{C} / \mathrm{N}$ but was less affected by $\mathrm{pH}$ and soil moisture. Norank-Xanthobacteraceae andnorank_Acidobacteriales had strong positive correlations with $\mathrm{pH}$ and soil moisture and weak correlations with altitude, total organic carbon, total nitrogen and C/N. For the fungi, Figure 4B shows that the first axis explained $35.27 \%$ of the variation, and the second axis explained $27.06 \%$, representing a cumulative explanatory power of $62.87 \%$. Of the top 10 fungal genera, unclassified_Basidiomycota andunclassified_Fungi showed strong positive correlations with total organic carbon, total nitrogen, moisture, $\mathrm{C} / \mathrm{N}$ and $\mathrm{pH}$, as didSebacina .

The heatmap results show that, for the bacteria, there was a very significant positive correlation between norank_Subgroup2 and soil moisture $(\mathrm{P}<0.01)$. There was a significant negative correlation between norank_Elsterales and pH (Figure 4C). OfAcidibacter, Acidothermus and Bryobacter, the three confirmed genera, Bryobacter had a significant negative correlation with $\mathrm{pH}$ and moisture $(\mathrm{P}<0.05)$, and Acidothermus showed a significant positive correlation with total organic carbon and total nitrogen. For the fungi, there was a significant positive correlation between Sebacina and total organic carbon and total nitrogen $(\mathrm{P}<0.05)$. There was a significant negative correlation between Saitozyma and soil pH (Figure 4D). The other microorganisms showed different degrees of positive and negative correlation with environmental factors, but none of these correlations were significant. The analysis results shown in the heatmap are consistent with the RDA.

FIGURE 4 Correlation analysis of the soil microbial community and environmental factors. A and B, RDA of the bacterial and fungal community at the genus level and environmental factors in rhizosphere soil. The different shapes of the points represent different families. The green and red arrows indicate the genus and the environmental factor, respectively. C-D, Analysis of the correlations between rhizosphere soil bacteria and fungi and environmental factors in the heatmap. Pearson coefficients were used to evaluate the correlations between environmental factors and selected genera (the 10 with the highest relative abundances). $\mathrm{R}$ values are shown in different colors in the diagram. For each genus, White, red and blue indicates no change, increased abundance, and decreased abundance, respectively. A, 1, norank_Subgroup2 ; 2,Acidothermus ; 3 , Acidibacter ; 4,Burkholderia-Caballeronia-Paraburkholderia ; 5, norank_Elsterales ; 6, Bryobacter ; 7, norank_Gemmataceae ; 8, norank_Xanthobacteraceae ; 9,Candidatus_Solibacter ; 10, norank_Acidobacteriales . B, 1, unclassified_Basidiomycota ; 2, unclassified_Fungi ; 3,Sebacina ; 4, Penicillium ; 5, Geminibasidium ; 
6,unclassified_Ascomycota ; 7, Archaeorhizomyces ; 8,Saitozyma ; 9, Russula ; 10, Tricholoma .

$4 \mid$

\section{DISCUSSION}

\section{1 | The relative abundance of rhizosphere soil microbial community changed}

At the phylum level, the difference in the carbon sequestration level of Masson pine families did not change the dominant phylum of soil bacteria, but the relative abundance of the bacterial phyla changed significantly. The dominant phyla mainly included Acidobacteria, Proteobacteria and Chloroflexi (Figure 2); this result is consistent with the results of previous studies (Bei, Moser, Wu, Müller, \& Liesack, 2019). The relative abundance of Acidobacteria increased with increasing carbon sequestration in the different families, and the differences among families reached significant and extremely significant levels (Figure 2). Acidobacteria are important soil microorganisms that play a very important role in the soil carbon cycle and in structuring ecosystems (Wang, et al., 2016). In this experiment, the total organic carbon content in the samples from the three families gradually increased with increasing carbon sequestration (Table 2), showing the same change trend as that of Acidobacteria. This result suggests that some members of Acidobacteria use soil carbon as the substrate for their metabolic activities and that the carbon content in the soil promotes this process. Previous research has also confirmed this view (Pankratov, et al., 2008). On the other hand, in our study, the relative abundance of Proteobacteria decreased with increasing carbon sequestration in the three families, although previous studies have shown that the relative abundance of Proteobacteria was associated with a higher carbon utilization rate (Fierer, Bradford, \& Jackson, 2007). Zheng (2014) explored the impact of ecological restoration on the structure of the soil bacterial community of mangroves (Rhizophora apiculata ) and found that the relative abundance of Proteobacteria was negatively correlated with the content of soil organic carbon. The phenomenon observed in our study may be due to the decisive influence of carbon sequestration in the Masson pine on Proteobacteria; there is evidence that the abovementioned vegetation had a greater impact on the bacterial taxa involved in soil carbon sequestration than other factors, such as soil environmental factors (Zhao, 2019). However, the specific mechanism of this influence and its process of action are still unclear and need to be further explored. Notably, Actinobacteria have been demonstrated to use mycelia to break down recalcitrant organic carbon (Dang, Yu, Le, Liu, \& Zhao, 2017), and Chloroflexi showed strong carbon sequestration (Li, 2019). However, the results of this study showed that the relative abundance of these two phyla did not change significantly with the increase in carbon sequestration in the different families (Figure $2 \mathrm{~A}$ ). We speculated that the cause of this phenomenon might be the low proportion of the two bacteria in the total rhizosphere community.

In terms of fungi, the dominant phyla mainly included Basidiomycota, Ascomycota, Mucoromycota and an unclassified phylum (Unclassified_k_functional). The relative abundance of Basidiomycota increased with the increase in the carbon sequestration level of the Masson pine families. The relative abundance of Ascomycota gradually decreased with increasing carbon sequestration, and the dominant rhizosphere fungi changed from Ascomycota to Basidiomycota (Figure 2, Figure S1). Wang et al. (2019) investigated the influence of grassland restoration in a Chinese pine (Pinus tabuliformis ) forest on fungi and found that when the carbon content in the soil decreased, the relative abundance of Ascomycota decreased significantly, and Basidiomycota replaced Ascomycota as the dominant fungal phylum; these results are consistent with the results of this experiment. Although the corresponding molecular mechanism is not fully understood, there is evidence that up to $30 \%$ of plant photosynthetic products can be transferred from plant roots to fungi (Gonzalez, et al., 2018; Nehls, Grunze, Willmann, Reich, \& Kuester, 2007). Moreover, some families of Basidiomycota have been confirmed to have enzymes that degrade complex macromolecular organic matter in the soil (Floudas, et al., 2012). When the soil carbon content increased, the abundance of Agaricomycetes significantly increased (Li, et al., 2020). Therefore, this might be the reason why Basidiomycota became the dominant fungus after the soil carbon content increased.

\subsection{Most members of the core microorganic community are involved in soil carbon metabolism}

Among the three Masson pine families with different carbon sequestration abilities, the core microorganisms 
in the rhizosphere soil were abundant. For bacteria, the core genera mainly includedBryobacter, Acidibacter , Acidothermus andBradyrhizobium (Figure 3). Among them, Acidothermus andBryobacter can directly utilize carbon in soil as a primary energy source and participate in the soil carbon cycle ( $\mathrm{Du}$, et al., 2017; Pankratov, et al., 2008; Kalyuzhnaya, Lidstrom, \& Chistoserdova, 2008). Therefore, with the increase in carbon sequestration in the Masson pine families, these genera both became core rhizosphere bacteria. On the other hand, both Acidibacter andBradyrhizobium are affiliated with Proteobacteria; their relative abundance declined with increasing carbon sequestration in the Masson pine families because these two genera are mainly involved in the nitrogen rather than the carbon metabolism process in soil ( Wang, Lin, Huang, Yang, \& Qu, 2019; Zhong, 2019; Liu, 2019). However, the carbon sequestration process in Masson pine had no significant impact on the nitrogen content of rhizosphere soil (Table 2). Therefore, both of these genera may have become core microbial genera simply because of their high overall abundance in soil, since Proteobacteria are common in almost all soil types (Zhang, \& Xu, 2008).

In terms of fungi, the core genera of the microorganism community were fewer, only Russula, Geminibasidium , Saitozyma andPenicillium (Figure S2). Because the structure of the fungal community was less stable than that of the bacterial community, the soil environment had a more obvious influence on the fungal community (Chen, et al., 2018; Xiao, Zhao, Yan, \& Guan, 2018). In this experiment, the core fungal genera were related to the carbon sequestration process in the Masson pine families to a certain extent. Studies have shown that carbon sequestration by plants stimulates the growth of Russulaand Geminibasidium and that these fungi regulate the uptake and accumulation of carbon by plants in a feedback system (Zhou, et al., 2011; Qiao, Zhou, Chai, Jia, \& Li, 2017). The relative abundance of Penicillium is mainly influenced by the soil carbon content $(\mathrm{Hu}, 2015 ; \mathrm{He}, 2016)$. The carbon content of the rhizosphere soil increased due to carbon sequestration by Masson pine (Table 2), which led toPenicillium becoming one of the core fungi in the microbial community.

\subsection{The soil properties affect the microorganic community}

Previous studies have shown that the composition of the soil microbial community is significantly correlated with soil nutrient levels (Tian, et al., 2017). Nitrogen limitation is common in most terrestrial ecosystems, and it often leads to intense competition between microorganisms and plants (Liu et al., 2016) as well as to changes in the classification and functional characteristics of the microbial community (Leff et al., 2015). The most typical changes observed in this study were decreases in the relative abundance of some rhizosphere fungal communities, such as Acidibacter and Bradyrhizobium(Figure S2). On the other hand, soil moisture is another important limiting factor in forests and has a strong influence on the soil bacterial community (Zhang, Liu, Xue, \& Wang, 2016), especially Proteobacteria (Figure 2, Table 2). Liu et al. (2020) found that the relative abundance of Proteobacteria in rhizosphere and nonrhizosphere soil under safflower (Carthamus tinctorius ) was significantly negatively correlated with soil moisture, which was similar to the results of this study. However, other studies have noted that the relative abundance of Proteobacteria in the nonrhizosphere soil of grassland vegetation (including Stipa bungeana and Artemisia vestita) was positively correlated with soil moisture (Liu, Huang, \& Zeng, 2016). This suggests that the effects of soil moisture on Proteobacteria might also be influenced by vegetation types and rhizosphere effects. However, the experimental results showed that soil moisture had little influence on fungi (Figure 4 B). Zhao et al. (2016) demonstrated that in semiarid grasslands, water restriction reduced the relative abundance of bacteria but did not change that of fungi, which increased the ratio of fungi to bacteria. Notably, pine forest soil has a higher carbon content than soils of other forest types, making it possible to identify fungal taxa that degrade low-quality substrates (Macdonald, et al., 2009). For instance, due to the lower substrate mass in coniferous forests, the diversity of Basidiomycetes and other lignin-degrading fungi was higher than that in prairie soils with lower carbon content (Allison, Hanson, \& Treseder, 2007). In this study, with the increase in the carbon content in soil, the relative abundance of Basidiomycetes also increased (Figure S1 B). Soil pH has been reported to play a key role in controlling microbial community composition (Cao et al., 2017). However, in this study, the effects of differences in soil pH among the different families were not significant; it is speculated that $\mathrm{pH}$ has little influence on the bacterial and fungal communities in Masson pine rhizosphere soil, except on genera that are relatively sensitive to $\mathrm{pH}$, such as Saitozyma (Figure $4 \mathrm{~B}, \mathrm{D}$ ). 
Notably, previous studies have shown that there is a strong relationship between rhizosphere microbes and plant root exudates (He, 2017). The changes in rhizosphere microbial communities among Masson pine families with different carbon sequestration capacities were discussed in this study, but the specific effects of rhizosphere exudates on soil microorganisms under these conditions were not analyzed. With the increase in carbon sequestration, was there a dominant substance that influenced the relative abundance of different rhizosphere bacteria or fungi? What were the patterns and mechanisms of the influence of these exudates? Were there any interactions between these secretions? These topics should be addressed in further research.

\section{5 | CONCLUSIONS}

In this study, there were significant differences among the soil rhizosphere microorganisms in samples from Masson pine families with different carbon sequestration abilities. With the increase in the carbon sequestration ability of the families, the dominant phylum of the soil rhizospheric bacteria changed from Proteobacteria to Acidobacteria, and the dominant phylum of fungi changed from Ascomycota to Basidiomycota. Compared with bacteria, fungi were more sensitive to carbon sequestration by Masson pine. On the other hand, by comparing the rhizosphere bacterial and fungal genera among samples from different families, we discovered a rich core bacterial and a fungal community, indicating the homogeneity of rhizosphere microbial diversity as the carbon sequestration activity of Masson pine varied. A significant proportion of the core microbiome was involved in carbon metabolism; these microorganisms might be better adapted to the acidic conditions of the native soil and respond more efficiently to root signals than other microorganisms. The composition and abundance of rhizosphere bacterial and fungal communities were greatly affected by the soil carbon content, soil moisture and altitude but were less affected by soil pH (exceptSaitozyma). This experiment provides early guidance for future in-depth research on the specific mechanisms and patterns of the soil microbial response to carbon sequestration by Masson pine.

\section{ACKNOWLEDGMENTS}

The research was financially supported by the National Key R\&D Program of China (2017YFD0600304) and the Priority Academic Program Development of Jiangsu Higher Education Institutions (PAPD).

We would like to thank Major biotechnology corporation (Shanghai, China) for assistance with sequencing services.

\section{DATA AVAILABILITY STATEMENT}

The data that support the findings of this study are available from the corresponding author upon reasonable request.

The data that supports the findings of this study are available in the supplementary material of this article.

\section{CONFLICTS OF INTEREST}

The authors declare no conflict of interest.

\section{REFERENCES}

Ainsworth, E. A. (2008). Rice production in a changing climate: a meta-analysis of responses to elevated carbon dioxide and elevated ozone concentration. Global Change Biology , 14(7), 1642-1650. https://10.1111/j.1365-2486.2008.01594.x

Allison, S.D., Hanson, C.A., \& Treseder, K.K. (2007). Nitrogen fertilization reduces diversity and alters community structure of active fungi in boreal ecosystems. Soil Biology \& Biochemistry , 39(8), 1878-1887. https://10.1016/j.soilbio.2007.02.001

Bei, Q., Moser, G., Wu, X., Müller, C., \& Liesack, W. (2019). Metatranscriptomics reveals climate change effects on the rhizosphere microbiomes in european grassland.Soil Biology \&3 Biochemistry , 138, 107604. https://10.1016/j.soilbio.2019.107604 
Cai, H.D.; Nong, S.Q.; Zhang, W.; Jiang, J.F.; Xiong, X.F.; \& Liu, F. (2014). Modeling of standing tree biomass for main species of trees in Guangxi Province. Forest Resources Management , 4, 58-66. https://10.13466/j.cnki.lyzygl.2014.04.012

Cao, C.Y.; Zhang, Y.; Qian, W.; Liang, C.P.; Wang, C.M.; \& Tao, S. (2017). Land-use changes influence soil bacterial communities in a meadow grassland in Northeast China. Solid Earth , 8(5), 1119-1129. https:// $10.5194 / \mathrm{se}-8-1119-2017$

Carrillo, Y.; Dijkstra, F.A.; Pendall, E.; LeCain, D.; \& Tucker, C. (2014). Plant rhizosphere influence on microbial $\mathrm{C}$ metabolism: the role of elevated $\mathrm{CO}_{2}, \mathrm{~N}$ availability and root stoichiometry.Biogeochemistry . 117(2-3), 229-240. https://10.1007/s10533-014-9954-5

Chen, H., \& Boutros, P.C. (2011). VennDiagram: a package for the generation of highly-customizable Venn and Euler diagrams in R.BMC Bioinformatics , 12, 35. https:// 10.1186/1471-2105-12-35

Chen, L., Xiang, W., Wu, H., Ouyang, S., Zhou, B., Zeng, Y., .. Kuzyakov, Y. (2018). Tree species identity surpasses richness in affecting soil microbial richness and community composition in subtropical forests. Soil Biology \& Biochemistry , 130, 113-121. https://10.1016/j.soilbio. 2018.12.008.

Cheng, W.; Zhang, Q.; Coleman, D.C.; Carroll, C.R.; \& Hoffman, C.A. (1996). Is available carbon limiting microbial respiration in the rhizosphere? Soil Biology \& Biochemistry , 28(10-11), 1283-1288. https://10.1016/S0038-0717(96)00138-1

Dang, P., Yu, X., Le, H., Liu, J., \& Zhao, Z. (2017). Effects of stand age and soil properties on soil bacterial and fungal community composition in chinese pine plantations on the Loess Plateau. PLoS One, 12(10), e0186501. https://10.1371/journal.pone.0186501

Du, S.Y., Yu, M., Liu, F.H., Xiao, L.L., Zhang, H.X., .. Chen, X. (2017). Effect of facility management regimes on soil bacterial diversity and community structure. Chinese Journal of Eco-Agriculture , 25(11), 1615-1625. https://10.13930/j.cnki.cjea.170291

Elias, M.; \& Potvin, C. (2003). Assessing inter- and intra-specific variation in trunk carbon concentration for 32 neotropical tree species. Canadian Journal of Forest Research , 33(6): 1039-1045. https://10.1139/x03-018

Feng, Y.Z.; Lin, X.G.; Mao, T.T.; \& Zhu, J.G. (2011). Diversity of aerobic anoxygenic phototrophic bacteria in paddy soil and their response to elevated atmospheric $\mathrm{CO}_{2}$. Microbial Biotechnology , 4(1): 74-81. https://10.1111/j.1751-7915.2010.00211.x

Fierer, N.; Bradford, M.A.; \& Jackson, R.B. (2007). Toward an ecological classification of soil bacteria. Ecology , 88(6), 1354-1364. https://10.1890/05-1839

Floudas, D.; Binder, M.; Riley, R.; Barry, K.; Blanchette, R.A.; Henrissat, B.; .. Hibbett, D.S. (2012). The paleozoic origin of enzymatic lignin decomposition reconstructed from 31 fungal genomes.Science , 336(6089), 1715-1719. https:// 10.1126/science.1221748.

Garcia-Lledo, A.; Vilar-Sanz, A.; Trias, R.; Hallin, S.; \& Baneras, L. (2011). Genetic potential for $\mathrm{N}_{2} \mathrm{O}$ emissions from the sediment of a free water surface constructed wetland. Water Research , 45(17), 56215632. https://10.1016/j.watres.2011.08.025

Gonzalez, E.; Pitre, F.E.; Page, A.P.; Marleau, J.; Nissim, W.G.; St-Arnaud, M.; ... Brereton, N.J.B. (2018). Trees, fungi and bacteria: tripartite metatranscriptomics of a root microbiome responding to soil contamination. Microbiome, 6, 53. https://10.1186/s40168-018-0432-5

He, F. (2016). The response of typical Quercus forest soil fungi and ectomycorrhizal fungi to environment factors and Cd. Master's Thesis, Jinan University, Jinan, China.

He, L.R. (2019). Overview of rhizosphere carbon cycle and microbial function under plant competition. Soil Science , 7(2), 154-160. https://doi.org/10.12677/HJSS.2019.72019 
$\mathrm{Hu}, \mathrm{J}$. (2015). The effects of grazing on soil nutrient and soil microbes in rhizosphere of Stipa grandis in the typical steppe. Doctor's Thesis, Chinese Academy of Agricultural Sciences, Beijing, China.

IPCC 2013 Climate Change. (2013). The Scientific Basis, Contribution of Working Group-I to the Fifth Assessment Report of Intergovernmental Panel on Climate Change (IPCC) . ON: Cambridge University Press

Jian, X.; Zhai, X.Y.; Wang, Y.; \& Cai, Y.Y. (2020). Influence of land use changes on soil total organic carbon and dissolved organic carbon in wetland. Acta Agriculturae Zhejiangensis , 32(3), 475-482. https://10.3969/j.issn. 1004-1524.2020.03.13

Jones, D.L.; Nguyen, C.; \& Finlay, R.D. (2009). Carbon flow in the rhizosphere: carbon trading at the soil-root interface. Plant Soil , 321(1-2), 5-33. https://10.1007/s 11104-009-9925-0

Kang, J.C. (2012). Wood properties variation analysis and superior families selection of Masson pine seedling seed orchard. Master's Thesis, Nanjing Forestry University: Nanjing, China.

Kahle, D.; \& Wickham, H. (2013). ggmap: Spatial Visualization with ggplot2. R Journal , 5(1), 144-161. https://10.1142/S0219025713500173

Kalyuzhnaya, M.G., Lidstrom, M.E., \& Chistoserdova, L. (2008). Real-time detection of actively metabolizing microbes by redox sensing as applied to methylotroph populations in lake washington. Isme Journal , 2(7), 696-706. https://10.1038/ismej.2008.32

Leff, J.W.; Jones, S.E.; Prober, S.M.; Barberan, A.; Borer, E.T.; Firn, J.L.; . . Fierer, N. (2015). Consistent responses of soil microbial communities to elevated nutrient inputs in grasslands across the globe.Proceedings of the national academy of sciences , 112(35), 10967-10972. https://10.1073/pnas.1508382112

Li, F.L.; Kong, Q.B.; Zhang, Q.; Wang, H.P.; Wang, L.M.; \& Luo, T. (2020). Spent mushroom substrates affect soil humus composition, microbial biomass and functional diversity in paddy fields. Applied Soil Ecology , 149, 103489. https:// 10.1016/j.apsoil.2019.103489

Li, K. (2019). Effects of Alternanthera philoxeroides on soil organic carbon and microorganisms, and the responses to nutrient and planting density. Master's Thesis, Shandong University, Jinan, China.

Liu, Q.Y.; Qiao, N.; Xu, X.L.; Xin, X.P.; Han, J.Y.; Tian, Y.Q.; .. Kuzyakov, Y. (2016). Nitrogen acquisition by plants and microorganisms in a temperate grassland. Scientific Reports , 6, 22642. https://10.1038/srep22642

Liu, W.W. (2019). Soil microbial composition and response of nitrogen and phosphorus soil enzymes to habitat in different stages of karst vegetation restoration. Master's Thesis, Guizhou University, Guiyang, China.

Liu, Y., Zhang, X., Yang, M.L., \& Wang, S.M. (2020). Study on the correlation between soil microbial diversity and ambient environmental factors influencing the safflower distribution in Xinjiang. Journal of Basic Microbiology , 60(6), 517-531. https://10.1002/jobm.201900626

Liu, Y.; Huang, Y.M., \& Zeng, Q.C. (2016). Soil bacterial communities under different vegetation types in the Loess Plateau.Environmental Science, 37(10), 3931-3938. https://10.13227 /j.hjkx.2016.10.035

Macdonald, C.A.; Thomas, N.; Robinson, L.; Tate, K.R.; Ross, D.J.; Dando, J.; \& Singh, B.K. (2009). Physiological, biochemical and molecular responses of the soil microbial community after afforestation of pastures with Pinus radiata . Soil Biology 83 Biochemistry , 41(8), 1642-1651. https://10.1016/j.soilbio.2009.05.003

Nehls, U., Grunze, N., Willmann, M., Reich, M., \& Kuester H. (2007). Sugar for my honey: carbohydrate partitioning in ectomycorrhizal symbiosis. ChemInform , 38(1), 82-91. https://10.1016/j.phytochem.2006.09.024

Ostle, N.; Whiteley, A.S.; Bailey, M.J.; Sleep, D.; Ineson, P.; \& Manefield, M. (2003). Active microbial RNA turnover in a grassland soil estimated using a $\left(\mathrm{CO}_{2}\right)$-C-13 spike. Soil Biology $\&$ Biochemistry, 35(7): 877-885. 
https://10.1016/S0038-0717(03)00117-2

Pankratov, T. A., Serkebaeva, Y. M., Kulichevskaya, I. S., Liesack, W., \& Dedysh, S.N. (2008). Substrateinduced growth and isolation of acidobacteria from acidic sphagnum peat. Isme Journal , 2(5), 551-560. https://10.1038/ismej.2008.7

Perez-Jaramillo, J.E.; de Hollander, M.; Ramirez, C.A.; Mendes, R.; Raaijmakers, J.M.; \& Carrion, V.J. (2019). Deciphering rhizosphere microbiome assembly of wild and modern common bean (Phaseolus vulgaris ) in native and agricultural soils from Colombia.Microbiome , 7, 114. https://10.1186/s40168-019-0727-1

Rodriguez, P.A.; Rothballer, M.; Chowdhury, S.P.; Nussbaumer, T.; Gutjahr, C.; \& Falter-Braun, P. (2019). Systems biology of plant-microbiome interactions. Molecular Plant , 12(6), 804-821. https:// 10.1016/j.molp.2019.05.006

Qiao, S.S., Zhou, Y.N., Chai, B.F., Jia, T., \& Li, C. (2017). Characteristics of fungi community structure and genetic diversity of forests in Guandi Mountains. Envionmental Science, 38(6), 2502-2512. https://10.13227 /j.hjkx.201611101

Schlesinger, W.H. (1990). Evidence from chronosequence studies for a low carbon-storage potential of soils. Nature, 348, 232-234. https://10.1038/348232a0

Schweinsberg-Mickan, M.S.Z.; Jorgensen, R.G.; \& Muller, T. (2012). Rhizodeposition: Its contribution to microbial growth and carbon and nitrogen turnover within the rhizosphere. Journal of Plant Nutrition and Soil Science, 175(5), 750-760. https://10.1002/jpln.201100300

Staddon, P.L.; Ramsey, C.B.; Ostle, N.; Ineson, P.; \& Fitter, A.H. (2003). Rapid turnover of hyphae of mycorrhizal fungi determined by AMS microanalysis of C-14. Science , 300(5622):1138-1140. https://10.1126/science.1084269

Tian, Q.; Taniguchi, T.; Shi, W.Y.; Li, G.Q.; Yamanaka, N.; \& Du, S. (2017). Land-use types and soil chemical properties influence soil microbial communities in the semiarid Loess Plateau region in China.Scientific Reports , 7, 45289. https://10.1038/srep45289

Wang, G.H., Liu, J.J., Yu, Z.H., Wang, X.Z., Jin, J., \& Liu, X.B. (2016). Research progress of Acidobacteria ecology in soils.Biotechnology Bulletin, 32(2), 14-20. https://10.13560/j. cnki.biotech.bull.1985.2016.02.002

Wang, L.J.; Deng, Y.H.; Zeng, X.P.; Liu, S.Z.; Meng, Z.; \& Li, Y.L. (2019). Water use characteristics of pioneer tree Pinus massoniana in south subtropical forest community in China.Journal of Central South University of Forestry $\&$ Technology, 39(3), 82-90. https://10.14067/j.cnki. 1673-923x.2019.03.014

Wang, K., Zhang, Y., Tang, Z., Shangguan, Z., Chang, F., Jia, F., ... Deng, L. (2019). Effects of grassland afforestation on structure and function of soil bacterial and fungal communities. Science of The Total Environment , 676, 396-406. https://10.1016/j.scitotenv.2019.04.259

Wang, S.S., Lin, G.Y., Huang, X.D., Yang, S.D., \& Qu, D. (2019). Effect of organic-inorganic compound fertilizer on diversity of bacteria in mulberry rhizosphere soil and endophytic bacteria in Root. Science of Sericulture , 45(4), 494-500. https://10.13441/j.cnki.cykx. 2019.04.004

Wu, F.; Sun, X.B.; Hu, X.F.; Zou, B.Z.; Lin, N.Q.; Lin, J.Q.; \& Ji, K.S. (2020). Response of nitrogen metabolism in Masson pine needles to elevated $\mathrm{CO}_{2}$. Forests. 11(4), 390. https://10.3390 /f11040390

Xiao, L.; Liu, G.B.; Li, P.; \& Xue, S. (2017). Elevated $\mathrm{CO}_{2}$ and nitrogen addition have minimal influence on the rhizospheric effects of Bothriochloa ischaemum. Science report , 7, 6527. https://10.1038/s41598-01706994-3

Xiao, W.Y.; Zhao, J.H.; Yan, X.L.; \& Guan, Q.W. (2018). Tree diversity determines the diversity of the taxonomic and functional structure of the fungal community in forest litter in southern China. Forest Science , 65(1), 40-47. https:// 10.1093/forsci/fxy037 
Xu, T. (2012). Effects of bamboo biochar on carbon-nitrogen-phosphorus and microbial diversity in pear orchard soil. Master's Thesis, Zhejiang University, Hangzhou, China.

Yin, P.Q.; Yin, M.Q.; Cai, Z.H.; Wu, G.Q.; Lin, G.H.; \& Zhou, J. (2018). Structural inflexibility of the rhizosphere microbiome in mangrove plant Kandelia obovata under elevated $\mathrm{CO}_{2}$. Marine Environmental Research , 140, 422-432. https://10.1016/j.marenvres. 2018.07.013

Zhang, C.; Liu, G.B.; Xue, S.; \& Wang, G.L. (2016). Soil bacterial community dynamics reflect changes in plant community and soil properties during the secondary succession of abandoned farmland in the Loess Plateau. Soil Biology \&3 Biochemistry , 97, 40-49. https://10.1016/j.soilbio.2016.02.013

Zhang, L. \& Xu, Z.H. (2008). Assessing bacterial diversity in soil. Journal of Soils $\mathscr{E}$ Sediments , 8(6), 379-388. https://10.1007/s11368-008-0043-Z

Zhang, W.; Fu, Y.; Li, J.F.; Meng, X.; \& Yang, J. (2015). Comparative study on Kjeldahl method and Dumas combustion method for total nitrogen measurement in soil. Chinese Agricultural Science Bulletin, 31(35), 172-175. https://CNKI:SUN:ZNTB.0.2015-35-029

Zhao, C.C.; Miao, Y.; Yu, C.D.; Zhu, L.L.; Wang, F.; Jiang, L.; .. Wan, S.Q. (2016). Soil microbial community composition and respiration along an experimental precipitation gradient in a semiarid steppe.Scientific Reports , 6, 24317. https://10.1038/srep24317

Zhao, P.Y. (2019). Assembly mechanism of soil microbial community in Larix principis-rupprechtii Mayr forest of subalpine north China in Shanxi Province. Doctor's Thesis, Institute of Loess Plateau, Taiyuan, China.

Zheng, Y. (2014). The effect of mangrove ecological-restoration on the seclected soil physical-chemical properties and community structures of benthic bacteria in the coastal wetlands of FuchengMaster's Thesis, Guangdong Ocean University, Guangdong, China.

Zhong, Z.M. (2019). Effects of Chamaecrista rotundifolia on dynamic changes of soil soluble nitrogen and bacterial communities in orchard red earth. Doctor's Thesis, Fujian Agriculture and Forestry University, Fuzhou, China.

Zhou, Z.Z., Chen, Y., Liang, K.N., Zhang, Y.C., Zhang, B., Huang, G.H., \& Ma, H.M. (2011). Screening study of mycorrhizal efficiently symbiosis of edible Russula funguswith host tree species. Journal of Central South University of Forestry \&3 Technology, 31(2), 7-14. https://10.14067 /j.cnki.1673-923x.2011.02.017

\section{SUPPORTING INFORMATION}

Additional supporting information may be found in the Additional file. 